PROCEEDINGS OF THE

AMERICAN MATHEMATICAL SOCIETY

Volume 136, Number 12, December 2008, Pages 4103-4110

S 0002-9939(08)08973-9

Article electronically published on July 17, 2008

\title{
ASYMPTOTIC DIMENSION OF FINITELY PRESENTED GROUPS
}

\author{
THANOS GENTIMIS
}

(Communicated by Alexander N. Dranishnikov)

\begin{abstract}
We prove that if a finitely presented group is one-ended, then its asymptotic dimension is greater than 1 . It follows that a finitely presented group of asymptotic dimension 1 is virtually free.
\end{abstract}

\section{INTRODUCTION}

The notion of asymptotic dimension of a metric space was introduced by Gromov in [6]. It is a large scale analog of topological dimension and it is invariant by quasiisometries. This notion has proved relevant in the context of Novikov's higher signature conjecture and it was investigated further by other people (see [1], 1], [9]).

In this paper we show the following:

Theorem 1. If $G$ is a one-ended finitely presented group, then $G$ has asymptotic dimension greater than or equal to 2 .

Also we deduce as a corollary:

Theorem 2. If $G$ is a finitely presented group with asdim $G=1$, then $G$ is virtually free.

For finitely generated groups the statement above doesn't hold. We give a counterexample at the end.

After we completed this work T. Januszkiewicz brought to our attention his joint paper with J. Swiatkowski ([7]), where they proved the same results independently.

\section{Preliminaries}

Metric spaces. Let $(X, d)$ be a metric space. If $A, B$ are subsets of $X$ we set $d(A, B)=\inf \{d(a, b): a \in A, b \in B\}$. A path in $X$ is a map $\gamma: I \rightarrow X$ where $I$ is an interval in $\mathbb{R}$. A path $\gamma$ joins two points $x$ and $y$ in $X$ if $I=[a, b], \gamma(a)=x$ and $\gamma(b)=y$. The path $\gamma$ is called an infinite ray starting from $x_{0}$ if $I=[0, \infty)$ and $\gamma(0)=x_{0}$. A geodesic, a geodesic ray, or a geodesic segment in $X$ is an isometry $\gamma: I \rightarrow X$ where $I$ is $\mathbb{R}$ or $[0, \infty)$ or a closed interval in $\mathbb{R}$. We use

Received by the editors August 15, 2005, and, in revised form, August 29, 2006.

2000 Mathematics Subject Classification. Primary 20F65, 20 F69.

Key words and phrases. Dimension theory, asymptotic dimension, finitely presented group.

Research supported by the program 'EPEAEK-Pythagoras' (75\% European grant, 25\% Greek national grant).

(C)2008 American Mathematical Society Reverts to public domain 28 years from publication 
the terms geodesic, geodesic ray, etc., for the images of $\gamma$ without discrimination. On a path-connected space $X$ given two points $x, y$ we define the path metric to be $\rho(x, y)=\inf \{$ length $(p)\}$ where the infimum is taken over all paths $p$ that join $x$ and $y$. A space is called a geodesic metric space if for every $x, y$ in $X$ there exists a geodesic segment which joins them. In a geodesic space the path metric is indeed a metric. A geodesic metric space $X$ is said to be one-ended if for every bounded $K, X-K$ has exactly one unbounded connected component. We say that $X$ is uniformly one-ended if for every $n \in \mathbb{R}^{+}$there is an $m \in \mathbb{R}^{+}$such that for every $K \subset X$ with $\operatorname{diam}(K)<n, X-K$ has exactly one connected component of diameter greater than $m$.

Groups. The Cayley graph of $G$ with respect to a generating set $S$ is the 1dimensional complex having a vertex for each element of $G$ and an edge joining vertex $x$ to vertex $x s$ for every vertex $x$ and every $s \in S$. The Cayley graph has a natural metric which makes it a geodesic metric space, where each edge has length 1 (see [9]). In fact any connected graph can be made a geodesic metric space in the same way.

We will use the same letter $G$ for both the group and its Cayley graph as a metric space. We also use van Kampen diagrams (see [8], chapter V, pgs. 236-240, and [4]). A van Kampen diagram $\mathfrak{D}$ for a word $w$ in $S$ representing the identity element of $G$ is a finite, planar, contractible, combinatorial 2-complex; its 1-cells are directed and labeled by generators and the boundary labels of each of its 2-cells are cyclic conjugates of relators or inverse relators. Further, the boundary label for $\mathfrak{D}$ is $w$ when read (by convention counterclockwise) from a base point in $\partial \mathfrak{D}$. We recall here that a word $w$ represents the identity element of $G$ if and only if the path in the Cayley graph labeled by $w$ is closed.

There is a natural map $f$ from the 1 -skeleton $\mathfrak{D}^{(1)}$ of $\mathfrak{D}$ to the Cayley graph of $G ; f$ sends the base point to a vertex $v$ of the Cayley graph and edges of $\mathfrak{D}^{(1)}$ to edges of the Cayley graph with the same label. Obviously $f$ is determined by the image of the base point, $v ; f$ is not necessarily injective. If we consider $\mathfrak{D}^{(1)}$ as a geodesic metric space giving each edge length 1 , then $d(x, y) \geq d(f(x), f(y))$ for every $x, y$ in $\mathfrak{D}^{(1)}$.

Finally we say that a group $G$ is virtually free if there exists a finite index subgroup $H$ of $G$ which is a free group.

Asymptotic dimension. A metric space $Y$ is said to be $d$-disconnected or that it has dimension 0 on the $d$-scale if there exist $B_{i} \subset Y$ such that

$$
Y=\bigcup_{i \in I} B_{i}
$$

with $\sup \left\{\operatorname{diam} B_{i}, i \in I\right\} \leq D<\infty, d\left(B_{i}, B_{j}\right) \geq d \forall i \neq j$.

Definition (Asymptotic Dimension 1). We say that a space $X$ has asymptotic dimension $n$ if $n$ is the minimal number such that for every $d>0$ we have $X=\bigcup X_{k}$ for $k=1,2, \ldots, n$ and all $X_{k}$ are $d$-disconnected. We then write $\operatorname{asdim} X=n$.

We say that a covering $\left\{B_{i}\right\}$ of $X$ has $d$-multiplicity $k$ if and only if every $d$ ball $B(x, d)$ in $X$ meets no more than $k$ sets $B_{i}$ of the covering. A covering has multiplicity $n$ if no more than $n+1$ sets of the covering have a nonempty intersection. A covering $\left\{B_{i}\right\}, i \in I$, is $D$-bounded if $\operatorname{diam}\left(B_{i}\right) \leq D \forall i \in I$. 
Definition (Asymptotic Dimension 2). We say that a space $X$ has $\operatorname{asdim} X=n$ if $n$ is the minimal number such that $\forall d>0$ there exists a $D$-bounded covering of $X$ with $d$-multiplicity $\leq n+1$.

The two definitions used here are the first two definitions Gromov gave in his paper [6]. It is not difficult to see that the two definitions are equivalent.

\section{MAIN THEOREM}

Before we get to the main theorem we will prove two lemmas that we will need below.

Lemma 1. Let $G$ be a finitely generated infinite group. Then $\operatorname{asdim} G>0$.

Proof. Let $\operatorname{asdim} G=0$ and fix $d>0$. Then according to the first definition we have that $G=X_{1}$ where $X_{1}=\bigcup B_{i}$ with:

(1) $\operatorname{diam} B_{i} \leq D, \forall i \in I$,

(2) $d\left(B_{i}, B_{j}\right) \geq d, \forall i \neq j$.

That means that $G$ is $d$-disconnected. Since $G$ is a connected graph it follows that we cannot have two distinct $B_{i}$ 's. So $G \subset B_{1}$, which means that $G$ is $D$-bounded. But since $G$ is finitely generated we have immediately that $G$ is finite, which is a contradiction.

Lemma 2. If $G$ is a one-ended finitely presented group, then $G$ contains a biinfinite geodesic.

Proof. Take any $n \in \mathbb{N}$. Then define

$$
C_{n}=\{\text { geodesic paths starting from } e \text { of length } n\} .
$$

We claim that:

a) $C_{n} \neq \emptyset$ for all $n \in \mathbb{N}$.

b) $C_{n}$ is a finite set for all $n \in \mathbb{N}$.

c) We can define $\pi_{n}: C_{n} \rightarrow C_{n-1}$ in a natural way.

a) Let $n_{0}$ in $\mathbb{N}$ be such that $C_{n_{0}}$ is empty. Then it is obvious that $G$ is a subset of $\bar{S}\left(e, n_{0}\right)$. To see this let $g \in G$ be such that $g \notin \bar{S}\left(e, n_{o}\right)$. Then $d(e, g)>n_{0}$. But then, since the Cayley graph of $G$ is a connected geodesic metric space, there exists a geodesic path $t$ connecting $e$ and $g$. So the length of that path is more than $n_{0}$. Obviously we can find a subpath $t^{\prime}$ of $t$ starting from $e$ with length $\left(t^{\prime}\right)=n_{0}$ (forget the last segments until the length becomes $n_{0}$ ). Thus by definition $t^{\prime} \in C_{n_{0}}$, which is a contradiction. So this means that $G \subseteq \bar{S}\left(e, n_{0}\right)$. Since $G$ is finitely generated and bounded it is obvious that $G$ is finite and

$$
|G| \leq \sum_{i=0}^{n_{0}} k^{i}=\frac{k^{n_{0}+1}-1}{k-1},
$$

where $k$ is the number of generators of the group. That is a contradiction since $G$ is one-ended, meaning that it has one infinite component. Thus we have proved (a).

b) All paths in $G_{n}$ are naturally inside $S(e, n)$. Inside that sphere we have at most

$$
\sum_{i=0}^{n} k^{i}=\frac{k^{n+1}-1}{k-1}
$$


vertices. Since our vertices are finite, our edges are finite also. Thus the paths we can construct with these finite edges are also finite, and finally the geodesic paths that we can have inside the ball $\bar{S}(e, n)$ are finite and this proves (b).

c) Consider

$$
\pi_{n}: C_{n} \rightarrow C_{n-1},
$$

which takes every geodesic path of length $n$ from $G_{n}$ and cuts off the last edge of that path. Then obviously what is left is also a geodesic and now the length is $n-1$. Thus it is contained in $C_{n-1}$. So for every $n \in \mathbb{N}, \pi_{n}$ is a natural well-defined map, proving (c).

So now consider the inverse limit sequence

$$
\{e\} \leftarrow C_{1} \leftarrow C_{2} \leftarrow \ldots \leftarrow C_{n} \leftarrow C_{n+1} \leftarrow \ldots
$$

such that every left arrow is a natural map $\pi_{n}$ for the appropriate index. By using the well-known statement that every inverse limit of compact, spaces exists and is actually compact, we obtain that there exists $F$ which is the inverse limit of the above sequence

$$
F=\lim _{\leftarrow}\left\{C_{i}, \pi^{i}\right\} .
$$

Now let $r \in F$. Obviously $r$ is a geodesic path for every $n \in \mathbb{N}$ and $r$ is infinite starting from $e$. Thus $r$ is an infinite geodesic ray starting from $e$.

Now consider

$$
K_{1}=\{g \in G \text { such that } d(g, e)=1\},
$$

which is all the vertices of $G$ that are a distance 1 from $e$, and let $L_{1}$ be all the edges that start from $e$. Also define $E_{1}$ to be all the geodesic rays starting from a point in $K_{1}$ and passing through $e$. Namely:

$$
E_{1}=\left\{r_{i}: r_{i} \text { are geodesic rays of } G, r_{i}(0) \in K_{1}, e \in r_{i}\right\} .
$$

Respectively define

$$
K_{n}=\{g \in G \text { such that } d(g, e)=n\}
$$

and

$$
E_{n}=\left\{r_{i}: r_{i} \text { are geodesic rays of } G, r_{i}(0) \in K_{n}, e \in r_{i}\right\} .
$$

Obviously $E_{1}$ can be seen as a subset of $F \times L_{1}$. Namely, if $Y_{\xi}$ is the subset of $E_{1}$ such that all geodesics start with the edge $\xi$ (which is in $L_{1}$ ), then

$$
E_{1}=\bigcup_{\xi \in L_{1}} Y_{\xi}
$$

Let $\xi=(e, s \cdot e)$. Then $Y_{\xi} \subseteq s \cdot F$.

Obviously $E_{1}$ is not empty. To see this let $r$ be the geodesic ray we defined starting from $e$. Let $(e, s e)$ be the first segment of $r$. Then define $s^{-1} \cdot r$. Since multiplying with an element doesn't change the respective distances, $s^{-1} \cdot r$ is also a geodesic starting from $s^{-1} \cdot e$ and passing through $s^{-1} \cdot s=e$. Thus $E_{1} \neq \emptyset$. With the same idea we prove that $E_{n} \neq \emptyset$ for every $n$. Thus if we take the inverse limit

$$
E=\lim _{\leftarrow} E_{n},
$$

we know that $E$ is nonempty and compact. Now let $k$ in $E$. Then $k$ is a path that passes through $e$, it is a geodesic line, and for every $n \in \mathbb{N}$ there exist exactly 2 points in $k$ such that the distance between them is $2 n$ and the distance of both of them from the base point $e$ is $n$. That $k$ is our bi-infinite geodesic line. 
Theorem 1. If $G$ is a one-ended finitely presented group, then asdim $G \geq 2$.

Proof. By Lemma 1 and since $G$ is one-ended we have that $G$ is infinite and thus $\operatorname{asdim} G>0$. We will show that $\operatorname{asdim} G \neq 1$. Let's suppose that $\operatorname{asdim} G=1$.

Let $M=\max \left\{\left|r_{i}\right|, i=1,2, \ldots, n: r_{i}\right.$ relation of $\left.G\right\}$, where $|r|=$ length of the word $r$. We fix $d>100 M+100$. Since asdim $G=1$ there is a covering $\mathbb{B}=\left\{B_{i}\right\}$ with

$$
G=\bigcup_{i \in I} B_{i}
$$

and diam $B_{i}<D, \forall i \in I$, such that every ball $B(x, d)$ intersects at most 2 sets of the covering $\mathbb{B}$. We may assume without loss of generality that if $r$ is a path in the Cayley graph labeled by a relator $r_{i}$, then $r$ is contained in some $B_{j} \in \mathbb{B}$.

Since $G$ is one-ended we have that $G$ has a bi-infinite geodesic $S$ (Lemma 2). Let $N=\max \left\{100 D^{100}, 300 M\right\}$. Choose an $x_{0} \in S$ and consider the ball $B\left(x_{0}, N\right)$ which separates the geodesic $S$ into two geodesic rays, $S_{1}$ and $S_{2}$. Since $G$ is oneended there is an $x$ in $S_{1}$, a $y$ in $S_{2}$, and a path $p$ with $p(0)=x$ and $p(t)=y$ such that $p \cap B\left(x_{0}, N\right)=\emptyset$.

We denote by $[x, y]$ the part of the geodesic $S$ that connects $x$ and $y$. Obviously length $([x, y]) \geq 2 N$. We denote by $w$ the path that corresponds to $[x, y] \cup p$.

We have then that

$$
\text { length }(w)=\text { length }([x, y])+\text { length }(p) \Rightarrow \text { length }(w)>200 D .
$$

So in order to cover the path $w$ we need at least 3 sets of the covering $\left\{B_{i}\right\}$.

We consider now the van Kampen diagram $\mathfrak{D}$ that corresponds to the path $w$ and the function $f$ from $\mathfrak{D}^{(1)}$ to the Cayley graph $G$. So $f(\partial \mathfrak{D})=w$. For notational convenience we label vertices and edges of $\partial \mathfrak{D}$ in the same way as $w$. So for example we denote the vertex on $\partial \mathfrak{D}$ which is mapped to $x_{0} \in w$ by $f$ also by $x_{0}$.

Let $B$ be a set of the covering that intersects $[x, y]$. We consider $f^{-1}(B)$. Let $C(B)$ be the union of all 2-cells of $\mathfrak{D}$ which have the property that their boundary is contained in $f^{-1}(B)$. Let $U$ be the collection of all such sets $B$ with the following property: For some connected component, $K$, of $C(B),[x, y] \cap K$ is contained in an interval $[a, b]$ with $a, b \in K$ such that $x_{0} \in[a, b]$. Let $d(K)=d(a, b)$ for such a component and let $d(B)$ be the maximal value of all $d(K)$ for the $K$ component of $C(B)$ such that $x_{0} \in[a, b]$. Let $B_{1}$ be a set in $U$ for which $d\left(B_{1}\right)$ is maximal. Let $K_{1}$ be the connected component of $C\left(B_{1}\right)$ for which $d\left(K_{1}\right)=d\left(B_{1}\right)$. Let's say that $K_{1} \cap[x, y]$ is contained in $\left[a_{1}, b_{1}\right]$ with $a_{1}, b_{1} \in K_{1}$. Let $e$ be the edge of $[x, y]$ adjacent to $a_{1}$ which does not lie in $[a, b]$. If $r$ is the 2 -cell containing $e$, there is some $B_{2} \in \mathbb{B}$ such that $C\left(B_{2}\right)$ contains $r$.

Let $C$ be the subset of $\mathfrak{D}$ which contains all 2-cells with boundary contained in $f^{-1}\left(B_{1} \cup B_{2}\right)$. Since $d\left(x_{0}, p\right) \geq N, C$ does not intersect $p$. Thus $\mathfrak{D}-C \neq \emptyset$. Let $K$ be the connected component of $C$ which contains $x_{0}$.

Let $P=\overline{\mathfrak{D}-K} \cap \bar{K} . P$ is connected since $K$ is connected. Each edge of $P$ is contained in two 2-cells. One of these 2-cells lies in $f^{-1}\left(B_{1} \cup B_{2}\right)$, and one does not lie in this set. It is not possible that all edges of $P$ are contained in a 2-cell of $f^{-1}\left(B_{2}\right)$. Indeed in this case we would have $d\left(B_{2}\right)>d\left(B_{1}\right)$, which is impossible. Since $r$ is contained in $C\left(B_{2}\right)$, some edge of $P$ is not contained in a 2-cell of $f^{-1}\left(B_{1}\right)$. It follows that there are 2 adjacent edges $e_{1}, e_{2}$ in $P$ such that one of them is contained in a 2-cell of $f^{-1}\left(B_{1}\right)$ and the other in a 2-cell of $f^{-1}\left(B_{2}\right)$. If $c$ is the 2-cell that contains $e_{1}$ and is not in $f^{-1}\left(B_{1} \cup B_{2}\right)$, then $c$ lies in a set 
$f^{-1}\left(B_{3}\right)$ with $B_{3} \in \mathbb{B}$ and $B_{3} \neq B_{1}, B_{2}$. The edges $e_{1}, e_{2}$ and the 2-cell $c$ have a vertex $v$ in common. So $v \in f^{-1}\left(B_{1} \cap B_{2} \cap B_{3}\right)$. It follows that $B_{1} \cap B_{2} \cap B_{3} \neq \emptyset$, a contradiction.

This concludes the proof.

Remark. The result above holds also for uniformly one-ended simply connected simplicial complexes. So if $X$ is a uniformly one-ended simply connected simplicial complex, then $\operatorname{asdim} X \geq 2$.

One can prove this in a similar way and we give a sketch here: Since $X$ is oneended, then $X$ has geodesic segments $\left[a_{n}, b_{n}\right]$ of length $n$ for every $n \in \mathbb{N}$. Using these segments and the fact that $X$ is uniformly one-ended, we can construct a closed path in $X$ like the path $w$ in the previous proof. Finally since $X$ is simply connected there is a map $f: D \rightarrow X$ with $f(\partial D)=w$, where $D$ is a disc. We may further assume that $f$ is simplicial by the simplicial approximation theorem. So this disc $D$ replaces the van Kampen diagram $\mathfrak{D}$ is the previous proof.

Of course the result does not hold for one-ended simply connected simplicial complexes; a half-line gives a counterexample. We remark finally that if a Cayley graph is one-ended, then it is uniformly one-ended.

We note that the following theorem holds:

Theorem (Dunwoody-Stallings [3). If $G$ is a finitely presented group, then $G$ is the fundamental group of a graph of groups such that all the edge groups are finite and all the vertex groups are 0 -ended or 1-ended.

Also it is known that:

Lemma 3. If all the vertex groups are 0-ended (i.e. finite), then $G$ is virtually free (see [10], page 120, Prop. 11).

Furthermore it is not difficult to prove the following lemma (see [5]):

Lemma 4. If $H<G$ and $H$ is finitely generated, then $\operatorname{asdim} G \geq \operatorname{asdim} H$.

Using the lemma above and Theorem 1 we have the stronger result:

Theorem 2. If $G$ is a finitely presented group with $\operatorname{asdim} G=1$, then $G$ is virtually free.

Proof. Let $G$ be a finitely presented group with asdim $G=1$. Let $\Gamma$ be the graph of groups of the Dunwoody-Stallings theorem. If a vertex group $H$ is one-ended, then from Theorem 1, asdim $H \geq 2$. But $H<G$, which means that asdim $G \geq 2$, which is a contradiction. So all vertex groups are 0 -ended. It follows that $G$ is virtually free.

Now we give an example of a finitely generated group which is not finitely presented, not virtually free and has asymptotic dimension 1 . Namely:

Proposition. Let $G=\mathbb{Z}_{2} \imath_{r} \mathbb{Z}$ be the wreath product of $\mathbb{Z}_{2}$ and $\mathbb{Z}$. Then $\operatorname{asdim} G=1$ and $G$ is not virtually free.

Proof. Since there exists a short exact sequence:

$$
0 \rightarrow\left(\oplus_{\mathbb{Z}} \mathbb{Z}_{2}\right) \rightarrow G \rightarrow \mathbb{Z} \rightarrow 0,
$$

by the Hurewicz type formula (see [2]) we have:

$$
\operatorname{asdim} G \leq \operatorname{asdim} \mathbb{Z}+\operatorname{asdim}\left(\oplus_{\mathbb{Z}} \mathbb{Z}_{2}\right) .
$$


Since every finitely generated subgroup $F$ of $\left(\oplus_{\mathbb{Z}} \mathbb{Z}_{2}\right)$ is finite, we have that all $F$ of that type have asymptotic dimension 0. Following the definition of asymptotic dimension for arbitrary discrete groups found in [2] we get:

$$
\operatorname{asdim}\left(\oplus_{\mathbb{Z}} \mathbb{Z}_{2}\right)=\sup \{\operatorname{asdim} F \mid F<G, \text { finitely generated }\}=0 .
$$

So we get:

$$
\operatorname{asdim} G \leq 0+1=1 .
$$

Since $G$ is a finitely generated infinite group, by Lemma 1 we have that asdim $G>0$. So $\operatorname{asdim} G=1$.

We will prove that $G$ is not virtually free. Let $G$ be virtually free. Then there exists an $S$ subgroup of $G$ such that $S$ is free and the index $|G: S|$ is finite. Let $N=N_{G}(S)$ be the normalizer of $S$ in $G$. Then $N=\left\{g s g^{-1}: g \in G\right.$ and $\left.s \in S\right\}$. We know that $N=N_{G}(S)$ is a normal subgroup of $G$ and that since $G: S$ is finite, $G: N$ is finite. Recall the short exact sequence

$$
0 \rightarrow\left(\oplus_{\mathbb{Z}} \mathbb{Z}_{2}\right) \rightarrow G \rightarrow \mathbb{Z} \rightarrow 0
$$

and denote the image of $\oplus_{\mathbb{Z}} \mathbb{Z}_{2}$ in $G$ to be $H$. Then obviously $H<G$, and we will prove that $N \cap H=\{e\}$. That is true since if $x \in N \cap H$, we will have that $x \in H$; thus there exists a $y$ in $\oplus_{\mathbb{Z}} \mathbb{Z}_{2}$ with $f(y)=x$. But since $o(y) \leq 2$ we have that $y^{2}=e$ in $\oplus_{\mathbb{Z}} \mathbb{Z}_{2}$, which gives us that $f(y)^{2}=e$ in $G$, meaning $x^{2}=e$ in $G$. But $x$ belongs to $N$; thus there exist $g \in G$ and $w \in S$ such that $x=g w g^{-1}$ and thus $x^{2}=g w^{2} g^{-1}=e$, which gives us $w^{2}=e$. Since $w$ belongs to $S$ and $S$ is free, we have that $w=e$. Thus $x=g e g^{-1}=e$, and we are done.

Now from the second theorem of isomorphisms and since $N$ is a normal subgroup of $G$, we have:

$$
\frac{H}{H \cup N} \simeq \frac{H \cdot N}{N}
$$

But $H \cup N=\{e\}$ and $\frac{H \cdot N}{N}<\frac{G}{N}$. Thus we get:

$$
H \simeq \frac{H}{H \cup N} \simeq \frac{H \cdot N}{N}<\frac{G}{N} .
$$

But that leads to a contradiction since $H$ is infinite and $\frac{G}{N}$ is finite.

\section{REFERENCES}

1. A. Dranishnikov, Asymptotic topology, Russian Math. Surveys 55 (2000), No. 6, 1085-1129. MR $1840358(2002 \mathrm{j}: 55002)$

2. A. Dranishnikov and J. Smith, Asymptotic dimension of discrete groups, Fundamenta Mathematicae 189 (2006), No. 1, 27-34. MR2213160(2007h:20041)

3. M. Dunwoody, The accessibility of finitely presented groups, Invent. Math. 81 (1985), No. 3, 449-457. MR807066 (87d:20037)

4. S. Gersten and Tim Riley, Filling length in finitely presentable groups, Geometriae Dedicata 92 (2002), 41-58. MR1934008 (2003i:20051)

5. M. Gromov, Hyperbolic groups, Essays in Group Theory (S. M. Gersten, ed.), MSRI Publ. 8, Springer-Verlag, 1987, pp. 75-263. MR919829 (89e:20070)

6. M. Gromov, Asymptotic invariants of infinite groups, Geometric Group Theory (G. Niblo, M. Roller, eds.), LMS Lecture Notes, vol. 182, Cambridge Univ. Press, 1993. MR 1253544 (95m:20041)

7. T. Januszkiewicz and J. Swiatkowski, Filling Invariants in Systolic Complexes and Groups, preprint (June 2005).

8. R. Lyndon and P. Schupp, Combinatorial Group Theory, Springer-Verlag, 1977. MR0577064 $(58: 28182)$ 
9. J. Roe, Lectures on Coarse Geometry, Univ. Lect. Series, Vol. 31, Amer. Math. Society, 2003. MR2007488 (2004g:53050)

10. J-P. Serre, Trees, Springer-Verlag, 1980. MR607504 (82c:20083)

11. G. Yu, The Novikov conjecture for groups with finite asymptotic dimension, Ann. of Math. 147 (1998), No. 2, 325-355. MR1626745 (99k:57072)

Department of Mathematics, University of Florida, Gainesville, Florida 32611

E-mail address: thanos@ufl.edu 